\title{
LARVAL TREMATODES (DIGENEA) OF THE GREAT POND SNAIL, lymnaea stagnalis (L.), (GAstropoda, Pulmonata) in Central Europe: A SURVEY OF SPECIES AND KEY TO THEIR IDENTIFICATION
}

\author{
FALTÝNKOVÁ A.*, NAŠINCOVÁ V.* \& KABLÁSKOVÁ L.*
}

\section{Summary:}

A survey of cercariae and metacercariae (Trematoda, Digenea) from the great pond snail (Lymnaea stagnalis) in Central Europe (Austria, Czech Republic, South-East Germany, Poland and Slovak Republic) is presented, based on a study of 3,628 snails examined from 1998 to 2005 . A total of 953 (26.3\%) L. stagnalis were infected with 24 trematode species comprising 19 species of cercariae and 11 species of metacercariae (six species occurred both as cercarie and metacercarie) of eight families. The dominant cercariae were those of Opisthioglyphe ranae (159 hosts infected), Plagiorchis elegans (141) (both family Plagiorchiidae) and Echinoparyphium aconiatum (153) (Echinostomatidae); 14 double infections were found. The most frequent metacercariae were those of Neoglyphe locellus (71) (Omphalometridae), E. aconiatum (66), Echinostoma sp. (59) and Moliniella anceps (48) (Echinostomatidae). In the previous studies carried out in Central Europe, a very similar spectrum of nine trematode families of 22 cercariae determined to species level and 43 types of cercariae reported under generic or provisional names, which can be in many cases conspecific with the previous taxa, were found. A simple key to identification of cercariae and metacercariae, together with their illustrations, is provided.

KEY WORDS : Trematoda, Mollusca, Basommatophora, cercariae, metacercariae, life cycle, prevalence, great pond snail, Lymnaea stagnalis.
Résumé : LARVES DE TRÉMATODES (Digenea) DE LYMNAEA STAGNALIS (L.), (Gastropoda, Pulmonata) en Europe Centrale : étude des ESPEECES ET CLÉ POUR LEUR IDENTIFICATION

Une étude des cercaires et métacercaires (Trematoda, Digenea) de l'escargot d'eau douce Lymnaea stagnalis en Europe Centrale (Autriche, République Tchéque, sud-est de l'Allemagne, Pologne et Slovaquiel a porté sur 3628 spécimens prélevés de 1998 à 2005. Un total de 953 (26,3%) L. stagnalis ont été retrouvés infecté par 24 espèces de trematodes, dont 19 espèces de cercaires et 11 de métacercaires (six espèces présentant à la fois des cercaires et des métacercaires) appartenant à huit familles. Les cercaires majoritairement présentes étaient celles d'Opisthioglyphe ranae (159 hôtes infestés), Plagiorchis elegans (141) (famille des Plagiorchiidae pour les deux) et Echinoparyphium aconiatum (153) (Echinostomatidae); 14 doubles infestations ont été trouvées. Les métacercaires majoritairement présentes étaient celles de Neoglyphe locellus (71) (Omphalometridae), E. aconiatum (66), Echinostoma sp. (59) et Moliniella anceps (48)

(Echinostomatidae). Les précédentes études menées en Europe Centrale ont rapporté des résultats proches: neuf familles de trématodes avec 22 cercaires identifiées en tant qu'espèces et 43 types de cercaires répertoriées sous des noms génériques ou provisoires, celles-ci pouvant dans de nombreux cas être conspécifiques avec les précédents taxa. Une clé d'identification des cercaires et des métacercaires avec leurs illustrations est fournie.

MOTS CLÉS : Trematode, Mollusque, Basommatophora, cercaire, métacercaire, cycle parasitaire, prévalence, escargot d'eau douce, Lymnaea stagnalis.

\section{INTRODUCTION}

T he great pond snail (Lymnaea stagnalis) is one of the best known aquatic molluscs with holarctic distribution, occurring in Europe, Asia, North Africa and North America (Jackiewicz, 2000; Beran, 2002; Glöer, 2002). In Europe, it is widely distributed and lives even in some parts of the Baltic sea with low salinity (Jackiewicz, 2000). It can be found predominantly in lower altitudes (150-250 m) where it inhabits slowly flowing or still waters, ponds, blind

\footnotetext{
* Faculty of Biological Sciences, University of South Bohemia \& Institute of Parasitology, Biology Centre, Academy of Sciences of the Czech Republic, Branišovská 31, 37005 Ceské Budějovice, Czech Republic

Correspondence: Anna Faltýnková.

Tel: +420-38-7775486 - Fax: +420-38-5310388.

E-mail: faltyn@paru.cas.cz
}

arms, pools and also periodic swamps, and it is able to survive short dry periods (Jackiewicz, 2000; Beran, 2002).

Being the most common and conspicuous snail, together with Planorbarius corneus (Planorbidae), L. stagnalis was examined in the very first larval trematode investigations of Nitzsch (1817), La Valette (1855), Pagenstecher (1857) and later Lühe (1909). Until today, L. stagnalis is still of interest, being the intermediate host not only of a wide variety of larval trematodes, which are among the most common species (Echinoparyphium aconiatum, Opisthioglyphe ranae, Diplostomum pseudospathaceum) in Central Europe (Faltýnková \& Haas, 2006), but also of the causative agents of cercarial dermatitis - cercariae of bird schistosomes (genus Trichobilharzia), which are studied quite intensively in Austria, Czech Republic, Germany and Poland (Allgöwer, 1990; Kolá řová et al 1997; Kolářová \& 
Horák, 1996; Konecny et al., 1999; Dvorák et al., 1999; Kalbe et al., 2000; Żbikowska, 2003, 2004; Sattmann et al., 2004; Horák \& Kolářová, 2005; Rudolfová et al., 2005; Hertel et al., 2006).

Loy \& Haas (2001) found 18 species of trematode larvae during their long-time investigation into the behaviour of cercariae from L. stagnalis in Germany. They found out that the spectrum did not change over the period of 20 years. The species composition was also similar to reports from the $19^{\text {th }}$ century (see references above). However, a substantial part of the cercariae found by Loy \& Haas (2001) were not determined to species. Cercariae with invalid or provisional names were often listed in articles dealing with the trematode fauna of $L$. stagnalis published in hardly accessible journals of regional importance. In some countries only few records of cercariae other than bird schistosomes exist (Konecny et al., 1999). Several attempts to compile an atlas of cercariae (Combes, 1980) or keys to identification of different types of cercariae (Odening, 1962a; Palm, 1966a, Blair, 1977) were made, but these are of difficult use. And recently, there is no updated and comprehensive information about larval trematodes of the great pond snail.

Because of the large size of the great pond snail, producing large amounts of cercariae over a long period, it is a favoured model organism for studies on parasitehost physiology, immunology and behaviour. However, correct identification of larval trematodes is the premise for any studies and also the knowledge of species composition is important for studies on the epidemiology of veterinary or medically important trematodes and for ecology of trematode communities. Therefore, a survey of larval trematodes in the great pond snail in Central Europe, based on original data from five countries and revised records from literature, is presented here, together with a simple key to identification suitable also for non-trematodologists and illustrations of the most common species. Detailed morphological descriptions of larval trematodes with more information about their distribution and life cycles will be presented in a separate monograph.

\section{MATERIAL AND METHODS}

\section{SAMPLING AND HANDLING OF SNAILS}

nails of Lymnaea stagnalis were sampled from April 1998 to October 2005 in still and slightly flowing water bodies of drainage areas of major rivers of Central Europe (Danube, Elbe, Main, Odra, Tisa). The regions studied comprised pond systems of South Bohemia and Moravia in the Czech Republic (see Faltýnková, 2005), in South-East Germany (see Faltýnková \& Haas, 2006), in the reserve of Milicz in Poland and ponds near Danube in Germany; blind arms of Danube in Austria and the Slovak Republic and channels along the Tisa River in East Slovakia (Fig. 1).

Molluscs $(n=3,628)$ were sampled with a strainer or hand picked on shores. In the laboratory, they were separated into glass containers with tap water $(100 \mathrm{ml})$. Shedding of cercariae was stimulated by light and heat for 4-6 hours. The internal morphology of cercariae and metacercariae was documented by drawings

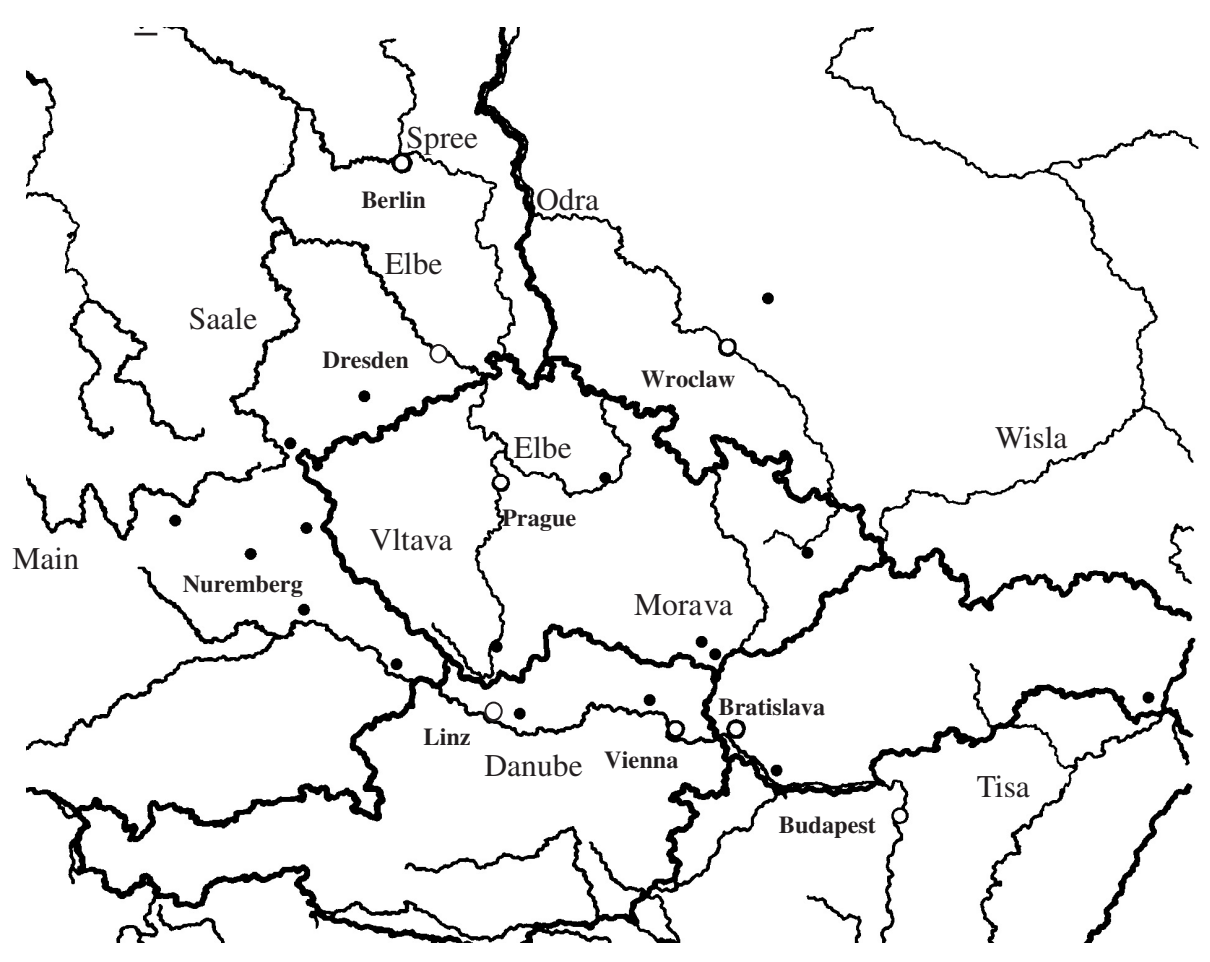

Fig. 1. - Survey of localities where samples of snails were taken. 
with aid of a drawing tube. Neutral red (to observe paraoesophageal glands of echinostomatids) and Nile blue were used for vital staining; urine solution was used to study the excretory system. For measurements, cercariae were fixed in hot $4 \%$ formaldehyde solution. Afterwards, a part of the molluscs were dissected to detect larval stages other than cercariae. Cercariae were identified with help of publications of Combes (1980), Niewiadomska (1986), Našincová (1992) and Niewiadomska et al. (1997). The system proposed by Gibson et al. (2002), Jones et al. (2005) and Bray et al. (2007) is followed.

\section{SURVEY OF LITERARY DATA}

To provide a complete survey of larval trematodes from Lymnaea stagnalis, literary data from Austria, Czech and Slovak Republics, Germany, Hungary and Poland were compiled to supplement the original data obtained during the present survey and in recent records of Faltýnková (2005) and Faltýnková \& Haas (2006). Records based on experimental infections were not considered. For better orientation, valid names of adult trematodes were provided together with original invalid or provisional names appearing in the literature.

A simple key to species of cercariae and metacercariae from L. stagnalis (Table II) is presented. Larval stages not identified at least to genus (Table III) are not included in the key, except for Cercaria spinulosa Ginetsinskaya, 1959. Only illustrations of common species recorded in the present study are presented; further figures can be found in publications of authors listed in Tables II and III.

\section{RESULTS}

I n the present survey, a total of $26.3 \%$ Lymnaea stagnalis were infected (Table I) with 24 trematode species comprising 19 species of cercariae and 11 species of metacercariae (six species occurred both as cercarie and metacercarie) of eight families (Table II). The dominant cercariae were those of Opisthioglyphe ranae (159 hosts infected), Plagiorchis elegans (141) (both Plagiorchiidae) and Echinoparyphium aconiatum
(153) (Echinostomatidae). The most frequent metacercariae were those of Neoglyphe locellus (71) (Omphalometridae), E. aconiatum (66), Echinostoma sp. (59) and Moliniella anceps (48) (Echinostomatidae). Metacercariae of O. ranae (3), P. elegans (1) and strigeids (1) were found less frequently.

In the previous studies carried out in Central Europe, as many as 22 cercariae determined to species level and 43 taxa recorded under generic or provisional names of nine families were reported from L. stagnalis (Tables II, III).

\section{KEY TO CERCARIAE}

1 Cercaria without tail (cercariaeum); intestinal caeca form cyclocoel Cyclocoelum microstomum

Cercaria with tail; intestinal caeca blind............ 2

2 Tail long, simple ................................................... 3

Tail forked (furcocercariae) ............................. 17

3 Cercaria with two suckers, ventral sucker more or less equatorial ........................................................ 4

Monostomous cercaria

Notocotylus attenuatus (Fig. 5A)

4 Collar and collar spines present; excretory granules (between oral and ventral sucker); excretory vesicle more or less spherical .............................................. 5

Collar and collar spines and excretory granules absent; stylet on dorsal part of oral sucker; excretory vesicle Y-shaped .................................... 12

5 Dorsal collar spines arranged in one row .............. 6 Dorsal collar spines in two rows; number of collar spines different 7

6 Cercaria large (body 400-500 $\mu \mathrm{m}$ long); excretory canals form finger-like processes anteriorly; excretory granules conspicuous; 47-50 collar spines

Cathaemasia hians (Fig. 3C-E)

Cercaria smaller; excretory canals form simple loops anteriorly; excretory granules small; 46-55 collar spines ....... Hypoderaeum conoideum (Fig. 4B, F) 7 Tail with fin folds; paraoesophageal glands may be present (stain intensively with neutral red) ............... 8

Tail without fin folds; no paraoesophageal glands....9

\begin{tabular}{|c|c|c|c|c|c|}
\hline \multirow[b]{2}{*}{ Country } & \multirow[b]{2}{*}{ Examined } & \multirow[b]{2}{*}{ Infected } & \multirow[b]{2}{*}{$\%$} & \multicolumn{2}{|c|}{ No. species } \\
\hline & & & & Cercariae & Metacercariae \\
\hline Austria & 203 & 22 & 10.8 & 3 & - \\
\hline Czech Republic & 1,078 & 409 & 37.9 & 14 & 7 \\
\hline Germany & 1,219 & 187 & 15.3 & 10 & - \\
\hline Poland & 669 & 273 & 40.8 & 13 & 2 \\
\hline Slovak Republic & 459 & 62 & 13.5 & 7 & 5 \\
\hline Total & 3,628 & 953 & 26.3 & 19 & 11 \\
\hline
\end{tabular}

Table I. - Survey of Lymnaea stagnalis examined. 


\begin{tabular}{lll}
\hline Valid name & Distribution & Original names and authorities
\end{tabular}

\section{Cyclocoelidae Stossich, 1902}

Cyclocoelum microstomum (Creplin, 1829)

Diplostomidae Poirier, 1886

*Diplostomum pseudospathaceum

Niewiadomska, 1984

Tylodelphys clavata (Nordmann, 1832)

Strigeidae Railliet, 1919

*Australapatemon burti (Miller, 1923)

Australapatemon minor (Yamaguti, 1933)

Cotylurus brevis Dubois et Rausch, 1950

Cotylurus cornutus (Rudolphi, 1808)

Schistosomatidae Stiles \& Hassall, 1898

*Trichobilharzia szidati Neuhaus, 1952

Sanguinicolidae von Graff, 1907

Sanguinicola inermis Plehn, 1905

Echinostomatidae Looss, 1899

"Echinoparyphium aconiatum Dietz, 1909

CZ, D, PL, SK

*Echinoparyphium recurvatum (Linstow, 1873)

*Echinostoma revolutum (Fröhlich, 1802)

*Hypoderaeum conoideum (Bloch, 1782)

*Isthmiophora melis (Schrank, 1788)

"Moliniella anceps (Molin, 1858)

*Paryphostomum radiatum (Dujardin, 1845)

Cathaemasiidae Fuhrmann, 1928

Cathaemasia hians (Rudolphi, 1809)

Notocotylidae Lühe, 1909

*Notocotylus attenuatus (Rudolphi, 1809)
$\mathrm{CZ} * *$

CZ, D, PL

CZ

CZ, D, PL

$\mathrm{CZ}$

CZ, D

CZ, D

A, CZ, D,

PL, SK

CZ, D

$\mathrm{CZ}, \mathrm{D}, \mathrm{PL}$

A, CZ, D, PL

CZ, D, PL, SK

CZ, D, PL

CZ, D

$\mathrm{CZ}, \mathrm{PL}$

CZ, PL

$\mathrm{A}, \mathrm{CZ}, \mathrm{D}, \mathrm{PL}$
Ždárská (1963)

Diplostomum spathaceum of Wisniewski (1958), Styczyńska-Jurewicz (1959), Ždárská (1963, 1964a), Odening (1964a) in part, Odening (1965a), Palm (1966a), Gottschalk (1971), Vojtek \& Vojtková (1971), Fleischerová (1972), Gelnar (1980); Furcocercaria sp. 1 of Odening (1962a); Cercaria sp. 1 of Bursian-Hartung (1965) in part; Niewiadomska (1986), Našincová (1992), Loy \& Haas (2001), Niewiadomska \& Laskowski (2002), Faltýnková (2005), Faltýnková \& Haas (2006), present study

Našincová (1992)

Apatemon burti (Miller, 1923) of Zajiček (1971), Našincová (1992); Apatemon gracilis of Zajíček \& Valenta (1964); Apatemon gracilis gracilis of Vojtek \& Vojtková (1971); Apatemon minor of Loy \& Haas (2001); Faltýnková \& Haas (2006), present results

Apatemon minor of Zajíček (1971), Našincová (1992)

Odening (1965a), Zajičcek (1971)

Ždárská (1963, 1964a), Odening (1965a), Zajî́ček (1971)

Cercaria ocellata La Valette, 1855 of Lühe (1909), Wisniewski (1958), Bertman \& Wojciechowska (1974), Farkaš (1976); Trichobilharzia ocellata of Emmel (1947), Ždárská (1963), Zajíček \& Valenta (1964) in part, Odening (1965a), Palm (1966a), Gelnar (1980), Našincová (1992) in part, Loy \& Haas (2001); Cercaria Trichobilharziae ocellatae of Bursian-Hartung (1965); Cercaria Trichobilharziae szidati of Dönges (1965); Trichobilharzia sp. König (2001); Neuhaus (1952), Kolářová et al. (1992) in part, Pilz et al. (1995), Kolářová \& Horák (1996) in part, Kolářová et al. (1997) in part, Dvořák et al. (1999), Sattmann et al. (2004), Rudolfová et al. (2005), Faltýnková (2005), Faltýnková \& Haas (2006); present study

Cercaria cristata La Valette, 1855 of Lühe (1909), Wisniewski (1958); Sanguinicola sp. (inermis) of Gelnar (1980); Scheuring (1922), Odening (1965a)

Cercaria echinata Siebold, 1837 of La Valette (1855), Lühe (1909), Zajičcek (1963), Bertman \& Wojciechowska (1974), Bertman (1980); Pseudechinoparyphium echinatum Kanev \& Vasilev, 1986 of Loy \& Haas (2001); Ždárská (1964a), Odening (1965a, 1965b), Gelnar (1980), Našincová (1992), Faltýnková (2005), Faltýnková \& Haas (2006), present study

Wisniewski (1958), Ždárská (1963, 1964a) in part, Odening (1965a, 1965b), Nezvalová (1970), Balúsek \& Vojtek (1973) in part, Gelnar (1980); Faltýnková (2005), Faltýnková \& Haas (2006), present study Cercaria echinostomi-revoluti of Zajíček (1963); Cercaria sp. 10 of Bursian-Hartung (1965); Wisniewski (1958), Ždárská (1963, 1964a) in part, Odening (1965a) in part, Gelnar (1980), Našincová (1992), Nezvalová (1970) in part, Kanev et al. (1993), Loy \& Haas (2001), Faltýnková (2005), Faltýnková \& Haas (2006), present study

Cercaria affinis Wesenberg-Lund, 1934 of Ahmed (1959); Odening (1965a), Nezvalová (1970), Balusek \& Vojtek (1973), Gelnar (1980), Našincová (1992), Loy \& Haas (2001), Faltýnková \& Haas (2006), present study

Euparyphium melis of Ždárská (1964b); Grabda-Kazubska \& Laskowski (1996), Loy \& Haas (2001), present study

Odening (1964b), Faltýnková (2005)

present study

Cercaria sp. 2 of Balüsek \& Vojtek (1973); Grabda-Kazubska et al. (1990)

Cercaria notocotyli-attenuati of Zajíček (1963); Cercaria monostomi Linstow, 1884 of Bursian-Hartung (1965), Bertman \& Wojciechowska (1974), Bertman (1980); Notocotylus sp. of Konecny et al. (1999); Ždárská (1963), Nezvalová (1970) in part, Balusek \& Vojtek (1973), Gelnar (1980), Našincová (1992), Faltýnková (2005), present study 
Plagiorchiidae Lühe, 1901

*Haematoloechus similis (Looss, 1899)

*Opisthioglyphe ranae (Fröhlich, 1791)

*Plagiorchis elegans (Rudolphi, 1802)

Plagiorchis laricola Skryabin, 1924

*Plagiorchis maculosus (Rudolphi, 1802)

Omphalometridae Odening, 1960

*Neoglyphe sobolevi Shaldybin, 1953

* Species found in recent studies.

**A - Austria, CZ - Czech Republic, D - Germany, PL - Poland, SK - Slovak Republic.

Table II. - Survey of species of cercariae reported from Lymnaea stagnalis in Europe.

\section{$\mathrm{CZ} \quad$ present study}

A, CZ, D, PL, Cercaria armata of Pagenstecher (1857); Cercaria armata Siebold of SK La Valette (1855), Lühe (1909); Xiphidiocercaria sp. 2 of Odening (1962b); Cercaria opisthioglyphe-ranae of Zajiček (1963); Cercaria sp. 7 of Bursian-Hartung (1965); Styczyńska-Jurewicz (1961), Žďárská (1963, 1964a) in part, Palm (1966b), Grabda-Kazubska (1969), Nezvalová (1970), Gelnar (1980), Dimitrov et al. (1989), Našincová (1992), Faltýnková (2005), Faltýnková \& Haas (2006), present study

A, CZ, D, PL Cercaria sp. 6 of Bursian-Hartung (1965); Styczyńska-Jurewicz (1961),

SK

Našincová (1992), Faltýnková (2005), Faltýnková \& Haas (2006), present study

CZ Z Ždárská (1966), Samnaliev et al. (1983), Gelnar (1980)

CZ Bušta (1987), Našincová (1992), Faltýnková (2005)

CZ, D, PL Našincová et al. (1989), Našincová (1992), present study
8 Cercaria large $(500-700 \mu \mathrm{m})$, with one large dorsoventral fin fold; tip of tail without contractile process; 35 collar spines; paraoesophageal glands absent

Moliniella anceps (Fig. 4C, G)

Cercaria small $(250-300 \mu \mathrm{m})$; with seven small, inconspicuous fin folds; tip of tail forms contractile process; 37 collar spines; 12 paraoesophageal glands ......... Echinostoma revolutum (Fig. 4A, E)

9 Cercaria with numerous small excretory granules; 37 or more collar spines .................................................. 11

Few large excretory granules; 27 collar spines ..... 10 10 Oral and ventral sucker possess crista; body smooth Paryphostomum radiatum (Fig. 4D, H)

Suckers without cristae; spinose body Isthmiophora melis 11 Cercaria large (500-600 um); 37 collar spines; angle spines larger Echinoparyphium aconiatum (Fig. 3F, G) Cercaria small $(200-250 \mu \mathrm{m}) ; 45$ collar spines of same length

Echinoparyphium recurvatum (Fig. $3 \mathrm{H}$ ) 12 Cercaria small $(100-120 \mu \mathrm{m})$; stylet straight, without anterior or posterior thickenings; penetration glands pre- and postacetabular

Haematoloechus similis (Fig. 5B, C)

Cercariae larger; stylet with anterior thickening (see Fig. 5E, G); penetration glands preacetabular

.. 13

13 Cercaria large $(270-320 \mu \mathrm{m})$, dark; stylet small, with incomplete basis and small anterior thickening; suckers of about same size ..... Opisthioglyphe ranae (Fig. 5D, E) Cercaria small $(130-200 \mu \mathrm{m})$; stylet conspicuous, with complete basis and larger anterior dilatation; oral sucker larger than ventral sucker .............. 14 14 Seven pairs of penetration glands ........................ 16
Seven penetration glands on one side and eight on the other 15 15 Stylet without thickening at its basis.

Plagiorchis elegans (Fig. 5F, G)

Stylet with thickening at its basis; posterior penetration glands paraacetabular ... Neoglyphe sobolevi (Fig. 5H, I) 16 Fat inclusions in body parenchyma; stylet without column Plagiorchis maculosus

No fat inlusions in body; stylet with column Plagiorchis laricola

17 Body with crista; pharynx absent (lophocercous cercaria) Sanguinicola inermis

Body without crista; pharynx present or within anterior organ ................................................ 18

18 Eye-spots with black pigment; five pairs of large penetration glands; furcae with fins

Trichobilharzia szidati (Fig. 3A, B)

Eye-spots colourless; different number of penetration glands; furcae without fins ..................... 19

19 Two pairs of preacetabular penetration glands ...... 20

Penetration glands postacetabular .................... 23

20 One row of hooked conspicuous spines on ventral sucker; flame cell formula $2[(2+2)+(2+(2))]=16 \ldots$ Tylodelphys clavata (Fig. 2G, H)

Three and more rows of small spines on ventral sucker; flame cell formula $2[(2+2)+(2+$ $2+(2))]=20$ 21 21 Cercaria with conspicuous large spines on whole body ............................................. Cercaria spinulosa

Body spinose except for oval area around ventral sucker .......................................................... 22

22 Cercaria with long oesophagus (longer than pharynx) .............................. Cotylurus cornutus (Fig. 2I)

Oesophagus short (as long as pharynx) Cotylurus brevis 


\begin{tabular}{|c|c|c|}
\hline Family/Generic name & Distribution & Original names and authorities \\
\hline \multicolumn{3}{|l|}{ Diplostomidae Poirier, 1886} \\
\hline Diplostomum sp. & $\mathrm{CZ}$ & $\begin{array}{l}\text { Diplostomum sp. (= Cercaria chromatophora Brown, 1931) of Zajíček \& Valenta } \\
\text { (1964) }\end{array}$ \\
\hline \multicolumn{3}{|r|}{ (1) 170} \\
\hline ?Australapatemon & $\mathrm{D}$ & Cercaria furcata Nitzsch, 1807 of Nitzsch (1817), La Valette (1855), Lühe (1909) \\
\hline Cotylurus sp. & $\mathrm{CZ}$ & $\begin{array}{l}\text { Cotylurus erraticus (Rudophi, 1809) of Zajǐček \& Valenta (1964), Vojtek \& Vojt- } \\
\text { ková (1971) }\end{array}$ \\
\hline Cotylurus sp. & $\mathrm{CZ}$ & $\begin{array}{l}\text { Cotylurus sp. II Ginetsinskaya, } 1959 \text { of Žďárská (1963), Opravilová (1969), Gelnar } \\
\text { (1980) }\end{array}$ \\
\hline ?Ichthyocotylurus sp. & $\mathrm{CZ}$ & Cercaria spinulosa Ginetsinskaya, 1959 of Zajiček \& Valenta (1964) \\
\hline \multicolumn{3}{|l|}{ Schistosomatidae Stiles \& Hassal, 1898} \\
\hline $\begin{array}{l}\text { Sanguinicolidae von Graff, } 1907 \\
\text { Sanguinicola spp. }\end{array}$ & Sanguinicolidae von Graff, 1907 & Konecny et al. (1999) \\
\hline \multicolumn{3}{|l|}{ Echinostomatidae Looss, 1899} \\
\hline Echinoparyphium ?recurvatum & $\mathrm{CZ}$ & Cercaria echinoparyphii sp. 1 of Zajíček (1963), Nezvalová (1970) \\
\hline ?Isthmiophora melis/?Paryphostomum sp. & $\mathrm{CZ}$ & Paryphostomum sp. 2 of Našincová (1992) \\
\hline Isthmiophora/Paryphostomum & $\mathrm{D}$ & $\begin{array}{l}\text { Cercaria fallax Pagenstecher, } 1857 \text { of Pagenstecher (1857), Lühe (1909), Bursian- } \\
\text { Hartung (1965) }\end{array}$ \\
\hline \multirow[t]{2}{*}{ ?Echinoparyphium aconiatum } & $\mathrm{CZ}$ & $\begin{array}{l}\text { Cercaria helvetica XXII Dubois } 1929 \text { of Nezvalová (1970); Echinostoma paraulum } \\
\text { Dietz, } 1909 \text { of Zajíček (1963), Nezvalová (1970), Gelnar (1980); Cercaria sp. } 1 \text { of } \\
\text { Balüsek \& Vojtek (1973) }\end{array}$ \\
\hline & $\mathrm{D}$ & Echinostome cercaria with 29 collar spines of Odening (1965b) \\
\hline Notocotylidae Lühe, 1909 & $\mathrm{CZ}$ & Notocotylus sp. Ginetsinskaya 1959 of Nezvalová (1970) in part \\
\hline \multicolumn{3}{|l|}{ Plagiorchiidae Lühe, 1901} \\
\hline ?Leptophallus & $\mathrm{CZ}$ & Cercaria helvetica VI Dubois, 1927 of Zajíček (1963) \\
\hline ?Leptophallus & $\mathrm{D}$ & Cercaria secunda Sinitzin, 1905 of Lühe (1909) \\
\hline "cf. Leptophallus sp. & PL & present study \\
\hline *Plagiorchis sp. 1 & $\mathrm{D}, \mathrm{SK}$ & present study \\
\hline *Plagiorchis sp. 2 & SK & present study \\
\hline \multirow[t]{15}{*}{ ?Plagiorchis sp. } & $\mathrm{CZ}$ & Cercaria helvetica IV Dubois 1927 of Zajíček (1963) \\
\hline & $\mathrm{CZ}$ & Cercaria helvetica XXVII Dubois 1929 of Žďárská (1963) \\
\hline & $\mathrm{CZ}$ & Cercaria limnaeae-ovatae Linstow 1884 of Ždárská (1963) \\
\hline & $\mathrm{D}$ & Cercaria tenuispina of Lühe (1909) \\
\hline & $\mathrm{CZ}$ & Xiphidiocercaria sp. 1 of Balusek \& Vojtek (1973), Gelnar (1980) \\
\hline & $\mathrm{CZ}$ & Xiphidiocercaria sp. 3 Balusek \& Vojtek, 1973 of Gelnar (1980) \\
\hline & $\mathrm{D}$ & Xiphidiocercaria sp. 3 of Odening (1962b) \\
\hline & $\mathrm{D}$ & Xiphidiocercaria sp. 4 of Odening (1962b) \\
\hline & $\mathrm{D}$ & Xiphidiocercaria sp. 5 of Odening (1962b) \\
\hline & $\mathrm{CZ}, \mathrm{D}$ & Xiphidiocercaria sp. A Palm, 1966 of Nezvalová (1970); Gelnar (1980) \\
\hline & $\mathrm{D}$ & Xiphidiocercaria sp. C Palm, 1966 of Palm (1966b) \\
\hline & & $\begin{array}{l}\text { Xiphidiocercaria I Ginetsinskaya } 1959 \text { of Palm (1966b), Nezvalová (1970), Gelnar } \\
\text { (1980) }\end{array}$ \\
\hline & $\mathrm{CZ}$ & Xiphidiocercaria II Ginetsinskaya 1959 of Žďárská (1963) \\
\hline & $\mathrm{CZ}, \mathrm{D}$ & $\begin{array}{l}\text { Xiphidiocercaria sp. III Ginetsinskaya } 1959 \text { of Nezvalová (1970); Palm (1966b); } \\
\text { Gelnar (1980) }\end{array}$ \\
\hline & $\mathrm{CZ}, \mathrm{D}$ & $\begin{array}{l}\text { Xiphidiocercaria sp. VI Ginetsinskaya, } 1959 \text { of Palm (1966b); Nezvalová (1970); } \\
\text { Gelnar (1980) }\end{array}$ \\
\hline Lissorchiidae Poche, 1926 & $\mathrm{D}$ & Asymphylodora sp. of Odening (1965a) \\
\hline ?Lissorchiidae & $\mathrm{D}$ & Cercariaeum sp. A of Palm (1967) \\
\hline \multirow[t]{3}{*}{ Species incertae sedis } & A, D & Unidentified echinostomous cercariae of Konecny et al. (1999), Loy \& Haas (2001) \\
\hline & A, D & Unidentified furcocercariae of Konecny et al. (1999), Loy \& Haas (2001) \\
\hline & $\mathrm{A}, \mathrm{D}$ & Unidentified xiphidiocercariae of Konecny et al. (1999), Loy \& Haas (2001) \\
\hline misidentified & PL & Cercaria auriculariae Zdun of Bertman \& Wojciechowska (1974) \\
\hline Strigeidae? & $\mathrm{D}$ & Cercaria brunnea Diesing, 1850 of Lühe (1909) \\
\hline furcocercaria & $\mathrm{D}$ & Cercaria fissicauda La Valette, 1855 of Lühe (1909) \\
\hline misidentified & $\mathrm{CZ}$ & Cercaria gracilis Wesenberg-Lund 1934 of Zajíček (1963) \\
\hline misidentified & PL & Cercaria longiremis Wesenberg-Lund, 1934 of Bertman (1980) \\
\hline misidentified & PL & Cercaria onusta Zdun of Bertman \& Wojciechowska (1974) \\
\hline misidentified & PL & Cercaria Petersen I Petersen of Bertman \& Wojciechowska (1974) \\
\hline misidentified & PL & Cercaria pseudogracilis Zdun of Bertman \& Wojciechowska (1974), Bertman (1980) \\
\hline misidentified & $\mathrm{CZ}, \mathrm{D}$ & Xiphidiocercaria sp.VII Odening 1962 of Nezvalová (1970) in part \\
\hline
\end{tabular}

Table III - Survey of species of cercariae identifited to genus or with provisional names reported from Lymnaea stagnalis in Europe. 


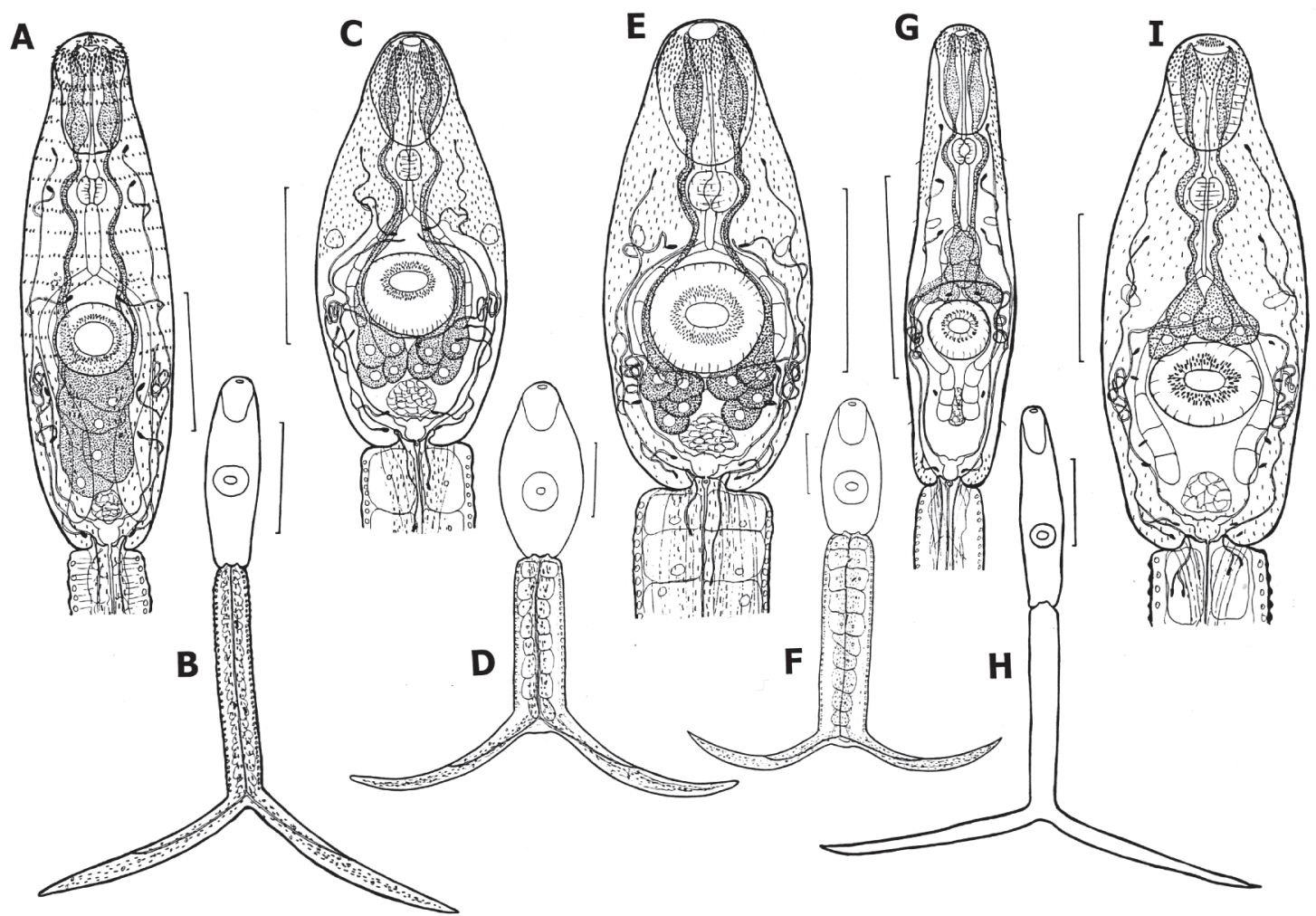

Fig. 2. - Diplostomum pseudospathaceum (A, B); Australapatemon burti (C, D); Australapatemon minor (E, F); Tylodelphys clavata (G, H); Cotylurus cornutus (I); A, C, E, G, I - detail of body, scale bars: A, C, E, I - 50 um, G - $100 \mu \mathrm{m}$; B, D, F, H - whole cercaria, scale bars: $\mathrm{B}, \mathrm{H}-100 \mu \mathrm{m}, \mathrm{D}, \mathrm{F}-50 \mu \mathrm{m}$.
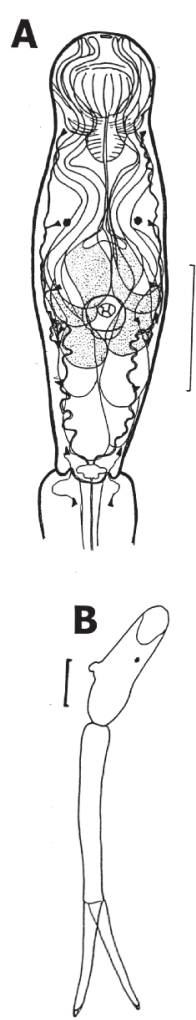
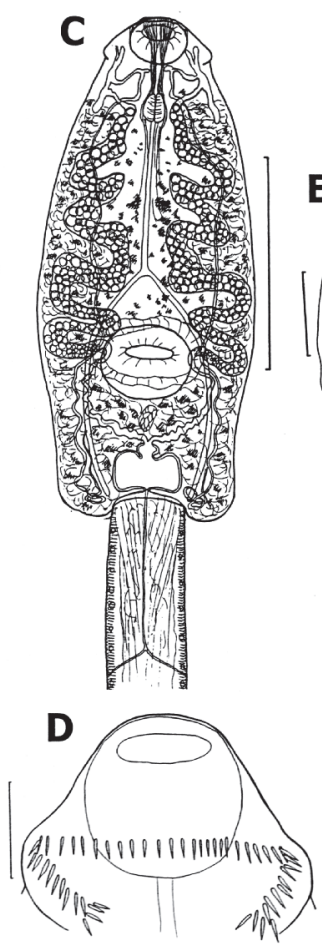
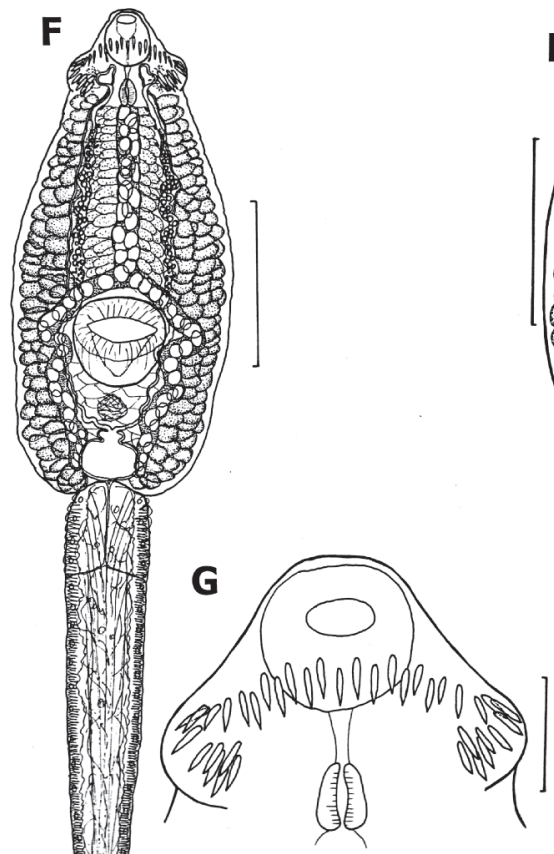

\section{H}

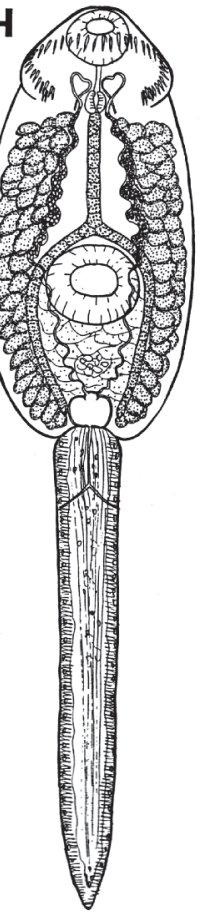

Fig. 3. - Trichobilharzia szidati (A, B); Cathaemasia hians (C, D, E); Echinoparyphium aconiatum (F, G); Echinoparyphium recurvatum $(\mathrm{H})$; A, C - detail of body, scale bars: A - $100 \mu \mathrm{m}, \mathrm{C}-200 \mu \mathrm{m}$; B, E, F, H - whole cercaria, scale bars: B, H - $100 \mu \mathrm{m}, \mathrm{E}, \mathrm{F}-200 \mu \mathrm{m}$; G, D - detail of anterior part with collar spines, scale bar: $50 \mu \mathrm{m}$. 
23 Two pairs of large postacetabular penetration glands; anterior organ with large conspicuous spines; caeca long, reach to body end; flame cell formula 2 [ $1+$ $1+1)+(1+1+1)+(2)]=16$

Diplostomum pseudospathaceum (Fig. 2A, B) Four pairs of small, poorly visible postacetabular penetration glands; anterior organ with fine spines; caeca short, reach to posterior end of ventral sucker; flame cell formula 2 [2 + $(2+$ $2+(1))]=14$

24 Body spined only on its anterior part Australapatemon burti (Fig. 2C, D) Whole body spinose Australapatemon minor (Fig. 2E, F)

\section{KEY TO METACERCARIAE}

1 Metacercaria spherical 2

Metacercaria oval 8

2 Cyst thick-walled; excretory granules and collar spines present; encysted in pericardium, kidney, less often in musculature

Cyst thin-walled; collar spines absent 6 3 Metacercaria large (about 270-380 $\mu \mathrm{m}$ in diameter)

A

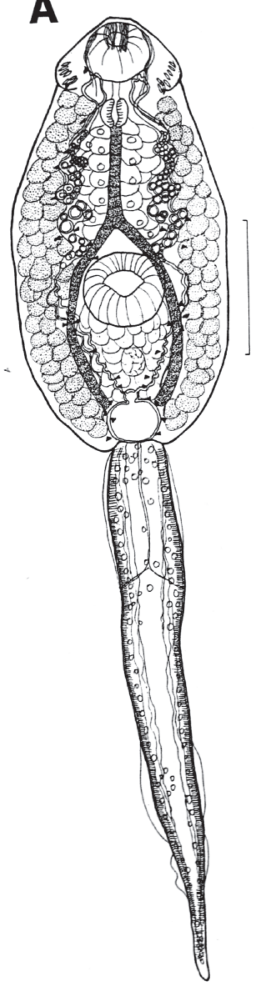

B

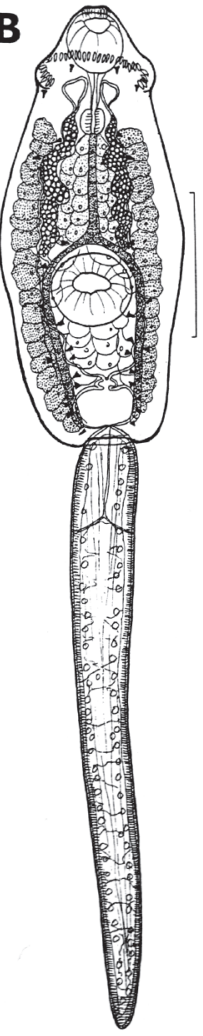

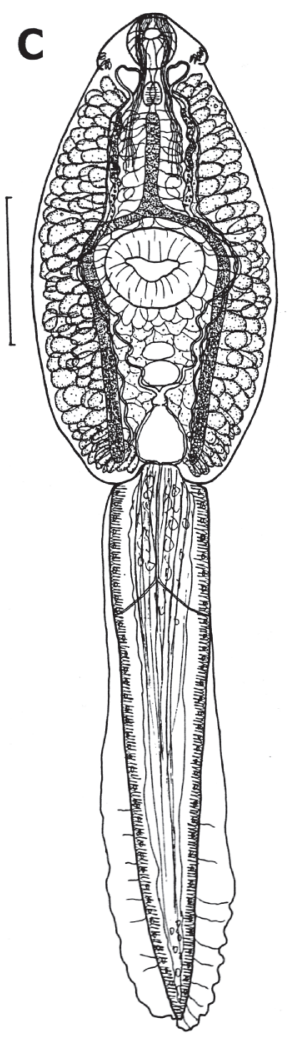

Metacercaria small (about $140 \mathrm{~mm}$ in diameter) .... 5 4 Collar of excysted metacercaria narrower than long body, with 35 spines of same length; three spherical anlagen of reproductive organs

Moliniella anceps (Fig. 6D)

Collar of excysted metacercaria of same width as short body; 37 collar spines, with conspicuous, larger angle spines

Echinoparyphium aconiatum (Fig. 6A)

5 Cercaria with 37 collar spines; excretory granules larger Echinostoma sp. (Fig. 6C)

Cercaria with 45 collar spines; excretory granules smaller ..... Echinoparyphium recurvatum (Fig. 6B) 6 Refractile granules in Y-shaped excretory vesicle; released stylet floating in cyst; anlagen of reproductive organs absent; encysted mainly in musculature ....... 7

Refractile granules and stylet absent; anlagen of reproductive organs present .......... Asymphylodora sp. 7 Metacercaria large $(200 \mu \mathrm{m})$, shape of stylet - see Fig. 5E Opisthioglyphe ranae (Fig. 6E)

Metacercaria small $(130 \mu \mathrm{m})$; shape of stylet - see Fig. $5 \mathrm{G}$ Plagiorchis sp. (Fig. 6F) 8 Cyst thin-walled; encysted in musculature (edge of mantle); Y-shaped transparent excretory vesicle

Neoglyphe locellus (Kossack, 1910) (Fig. 6G)
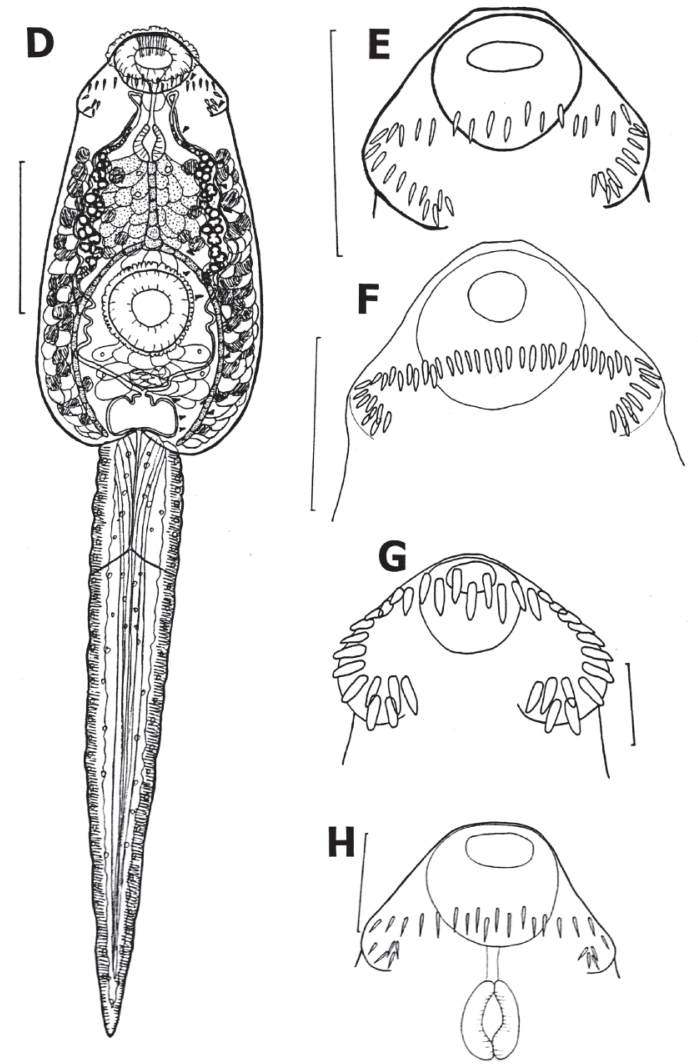

Fig. 4. - Echinostoma revolutum (A, E); Hypoderaeum conoideum (B, F); Moliniella anceps (C, G); Paryphostomum radiatum (D, H); AD - whole cercaria, scale bars: A, B, D - $100 \mu \mathrm{m}, \mathrm{C}-200 \mu \mathrm{m}$; E-H - detail of anterior part with collar spines, scale bar: $50 \mu \mathrm{m}$. 

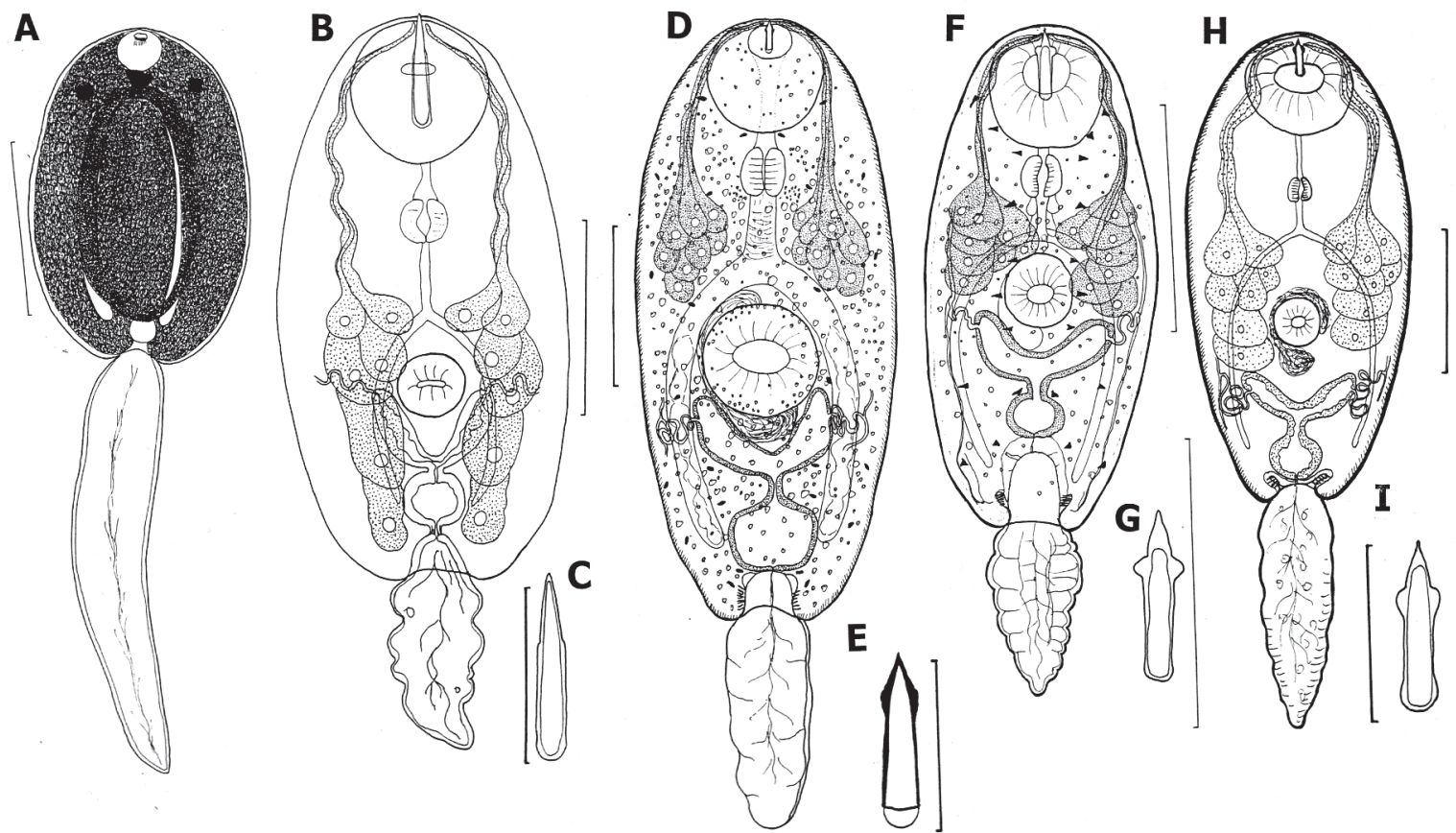

Fig. 5. - Notocotylus attenuatus (A); Haematoloechus similis (B, C); Opisthioglyphe ranae (D, E); Plagiorchis elegans (F, G); Neoglyphe sobolevi $(\mathrm{H}, \mathrm{I}) ; \mathrm{A}, \mathrm{B}, \mathrm{D}, \mathrm{F}, \mathrm{H}$ - whole cercaria, scale bars: A - $200 \mu \mathrm{m}, \mathrm{B}-50 \mu \mathrm{m}, \mathrm{D}, \mathrm{F}, \mathrm{H}-100 \mu \mathrm{m}$; C, E, G, I - stylet, scale bars: C, E, I - $30 \mu \mathrm{m}$, $\mathrm{G}-50 \mu \mathrm{m}$.

\section{DISCUSSION}

Tn Lymnaea stagnalis a total of 24 trematode species comprising 19 species of cercariae and 11 species of metacercariae of eight families were found

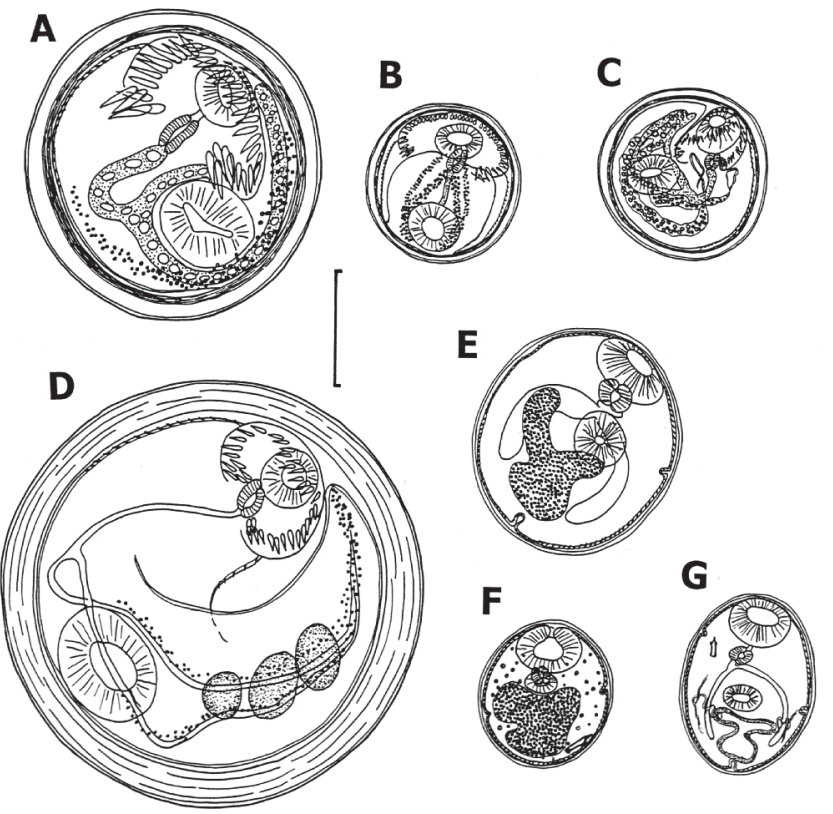

Fig. 6. - Echinoparyphium aconiatum (A), Echinoparyphium recurvatum (B), Echinostoma sp. (C), Moliniella anceps (D), Opisthioglyphe ranae (E), Plagiorchis sp. (F), Neoglyphe locellus (G); scale bar $100 \mu \mathrm{m}$. recently. A rather similar fauna of 22 cercariae determined to species level and 43 cercariae reported under generic or provisional names of nine trematode families were found in former studies carried out in Central Europe.

However, it must be assumed that the real number of existing species is almost certainly much lower than the latter number of 43 taxa, because some species are most probably conspecific with the determined ones. Especially xiphidiocercariae (Plagiorchiidae) with quite similar morphological characteristics were reported under several provisional names; in Table III trematodes with insufficient, dubious or missing descriptions were included. Unfortunately, the real identity of these records cannot be worked out because of insufficient or lacking descriptions. In the group of Species incertae sedis, mainly cercariae obviously misidentified without any descriptions were included, because they were assigned to species being specific only for planorbid snails that never occur in lymnaeids.

The dominant cercariae were those of Opisthioglyphe ranae, Echinoparyphium aconiatum and Plagiorchis elegans of the families Plagiorchiidae and Echinostomatidae. These three species are widely distributed in Europe and were found as the most frequent in all countries where samples were undertaken.

The most frequent metacercariae were those of $\mathrm{NeO}$ glyphe locellus (Omphalometridae), with cercariae being most abundant in Planorbarius corneus (Planorbidae). The second most common were metacercariae of the 
family Echinostomadidae: E. aconiatum, Echinostoma sp. and Moliniella anceps, which often occurred together. This family contains most species encysting in molluscs. Metacercariae of plagiorchids were found less frequently, since Plagiorchis elegans is reported to use snails accidentally. However, the common Opisthioglyphe ranae is known to encyst in molluscs. The strigeid metacercariae could belong to a species of Cotylurus, which encyst frequently in molluscs (Našincová, 1992).

The highest species diversity of cercariae in L. stagnalis was found in the pond systems of the Czech Republic and Poland. The number of species found in Germany was slightly lower, but the spectrum of cercariae (18 species) in the same region found by Loy \& Haas (2001), was not much higher. In Austria and the Slovak Republic more blind arms of the Danube river than ponds were examined and lower numbers of L. stagnalis could be sampled.

Only one species of the family Strigeidae, Australapatemon burti, was found in the present study. The genus Australapatemon, established by Sudarikov (1959), was regarded as a subgenus of Apatemon by Dubois (1968). For a long time the species of Australapatemon were reported as Apatemon gracilis (as listed in Table II), although the cercariae of Australapatemon differ in morphology and biology from those of Apatemon (long vs. rudimentary caeca, different flame cell formula and body spination, metacercariae in leeches vs. fishes, Našincová 1992). No cercariae of Cotylurus were found in this study, although several species were recorded mainly by the Czech authors (Tables II, III). Cotylurus erraticus (Rudolphi, 1819) (transferred to Ichthyocotylurus) was erroneously identified by Zajíček \& Valenta (1964). Their species actually belongs to the genus Cotylurus and probably represents a separate species (Table III). It could not be assigned to any other known species of Cotylurus because of a different type of body spination (spination the same as in C. cornutus except for an aspinose part in the area of the excretory vesicle).

Schistosome cercariae occurring in L. stagnalis were identified as Trichobilharzia szidati, although Rudolfová et al. (2005) found another cryptic species in this snail host (listed in Table III). The prevalence of T. szidati was very similar in most countries (Czech Republic, Germany and Poland). It was not found in Austria, presumably because of a short sampling period. Nearly the same species composition of echinostomes as in previous surveys was found in the present study. Cercariae with cristae on suckers were considered as Paryphostomum radiatum and those without cristae could be Isthmiophora melis (Table II). Grabda-Kazubska \& Laskowski (1996) redescribed the life cycle of I. melis and found that it is difficult to separate these two species, even with the aid of chaetotaxy. However,
$P$. radiatum has a crista on the oral and ventral sucker (Našincová et al., 1993), which could be considered as a distinguishing feature, and the species characterized by Našincová (1992) as Paryphostomum sp. without cristae could be I. melis. Grabda-Kazubska \& Laskowski (1996) noted that Cercaria fallax Pagenstecher, 1857 was very probably I. melis.

The spectrum of plagiorchiid species was also very similar as in former surveys, except for Haematoloechus similis, reported only from planorbid snails. The species of Plagiorchis elegans and P. laricola were both included in Table II, although it is still unclear if they are separate species or not (Našincová, 1992). Since there exist features to distinguish them, they were also included in the key, although the characters (number of penetration glands, column in stylet) are difficult to observe.

It can be assumed that the trematodes from L. stagnalis are rather well known and their distribution exceeds the area of Central Europe. The same species were found for example in Great Britain (Khan, 1960) or Finland (Niewiadomska et al., 1997); this occurrence is connected with the large distribution area of the great pond snail in Europe.

\section{ACKNOWLEDGEMENTS}

The authors are deeply indebted to the following persons (in alphabetic order): Bernardett Beran, Marta Bombarová, Jennifer Borelli, Martina Borovková, Sebastian Brachs, Oleg Ditrich, Vlado Dudiňák, Wilfried Haas, Jan Hertel, Alexander Holweg, Roman Kuchta, Dennis Kallert, Christina Loy, Mikuláš Oros, Sabine Ponader, Irena Šetlíková, Blanka Škoríková, Marta Špakulová, Romana Vlčková, J. Witkowski, Cynthia Wulff, Elzbieta Żbikowska and Janusz Zbikowski for help with field collections; thanks are also due to Tomáš Scholz and Aneta Kostadinova for valuable comments on the manuscript. This study was supported by the Grant Agency of the Academy of Sciences of the Czech Republic (project No. IAA6022404), the Grant Agency of the Czech Republic (projects No. 524/ 03/H133; 524/07/P086), research projects of the Institute of Parasitology, AS CR (Z60220518 and LC522) and Deutsche Bundesstiftung Umwelt.

\section{REFERENCES}

Ahmed Z. Die Cercarienfauna der Umgebung von Münster (Westf.) und der experimentell ermittelte Individualcyclus von Echinoparyphium spiniferum La Valette (Trematoda). Zeitschrift für Parasitenkunde, 1959, 19, 67-99.

Allgöwer R. Zur Trematodenfauna einiger Freiburger Baggerseen, mit besonderer Berücksichtigung des Erregers der 
Zerkariendermatitis beim Menschen. Mitteilungen bad. Landesvereins für Naturkunde und Naturschutz N.F., 1990, 15, 59-79.

BAüseK J. \& Vojtek J. [Contribution to the knowledge of our cercariae.] Folia Facultatis Scientiarum Naturalium Universitatis Purkynianae Brunensis, 1973, 14, 3-119 (in Czech).

Beran L. Aquatic molluscs of the Czech Republic, distribution and its changes, habitats, dispersal, threat and protection, Red list. Sbornik Prírodovedného klubu v Uherském Hradišti, 2002, Supp., 10, 258 p. (in Czech).

Bertman M. [Seasonal dynamics of infestation of the great pond snail (Lymnaea stagnalis L.) in fishponds in the reserve "Stawy Milickie".] Wiadomosci Parazytologiczne, 1980, 26 (1), 23-29 (in Polish).

Bertman M. \& WojciechowsKa K. [Fauna of cercariae in snails from freshwater reservoirs by Wroclaw and the surroundings.] Przeglad Zoologiczny, 1974, 18 (3), 354-359 (in Polish).

BLAIR D. A key to cercariae of British strigeoids (Digenea) for which the life-cycles are known, and notes on the characters used. Journal of Helminthology, 1977, 51, 155-166.

Bray R.A., Jones A. \& Gibson D.I. Keys to the Trematoda. Volume 3. CABI Publishing Wallingford \& Natural History Museum, London, 2007 (in press).

Bursian-Hartung G. Untersuchungen über die Cercarienfauna des Diskauer Teichgebietes bei Halle. Hercynia, 1965, 2, 63-127.

BušTa J. Chaetotaxy of the cercaria of Plagiorchis maculosus (Rudolphi, 1802) (Trematoda, Plagiorchiidae). Folia Parasitologica, 1987, 34, 219-224.

Combes C. Atlas Mondial des Cercaires. Mémoires du Muséum National d'Histoire Naturelle, Série A, Zoologie, 1980, 115, $5-235$.

Dimitrov V., Bušta J. \& Kanev I. Chaetotaxy of cercariae of Opisthioglyphe ranae (Frölich, 1791) (Trematoda: Plagiorchidae). Folia Parasitologica, 1989, 36, 265-274.

DÖNGES J. Schistosomatiden-Cercarien Süddeutschlands. Zeitschrift für Tropenmedizin und Parasitologie, 1965, 16, 305-321.

Dubors G. Synopsis des Strigeidae et des Diplostomatidae (Trematoda). Mémoires de la Société Neuchateloise des Sciences Naturelles, 1968, 10, 1-261.

Dvořák J., Sattmann H., Konecny R. \& Horák P. Larval stages of avian schistosomes in Austria: recent data from a Czech-Austrian cooperation project. Mitteilungen der Österreichischen Gesellschaft für Tropenmedizin und Parasitologie, 1999, 21, 69-76.

EMmel L. Beiträge zur Biologie und Morphologie der Cercaria ocellata. Zentralblatt für Bakteriologie, Parasitenkunde, Infektionskrankheiten und Hygiene, 1947, 152, 285-291.

FALTÝNKOVÁ A. Larval trematodes (Digenea) in molluscs from small water bodies near České Budějovice, Czech Republic. Acta Parasitologica, 2005, 50, 49-55.

FALTÝNKOVÁ A. \& HAAS W. Larval trematodes in freshwater molluscs from the Elbe to Danube rivers (South-East Germany): before and today. Parasitology Research, 2006, 99, 572-582.
FARKAŠ J. [On the problems of cercarial dermatitis.] Ceskoslovenská Dermatologie, 1976, 51 (1), 248-254 (in Slovak).

Fleischerová J. [Contribution to the knowledge of intermediate hosts of the trematode Diplostomum spathaceum (Rud., 1891) Braun 1893.] Folia Facultatis Scientiarum Naturalium Universitatis Purkynianae Brunensis, 1972, 13 (36), 21-29 (in Czech).

GELNAR M. Taxonomy and morphology of developmental stages of trematodes. Msc Thesis, Faculty of Sciences, University of J.E. Purkyně in Brno, 1980, 189 p. (in Czech).

Gibson D.I., JONES A. \& Bray R.A. Keys to the Trematoda. Volume 1. CABI Publishing Wallingford \& Natural History Museum, London, 2002.

GLÖER P. Die Süßwassergastropoden Nord- und Mitteleuropas. Bestimmungschlüssel, Lebensweise, Verbreitung. Die Tierwelt Deutschlands, 2002, 73, 1-327.

GotTschalk C. Untersuchungen über fischpathogene Cercarien in Fischgewässern der Oberlausitz und Thüringens. Parasitologische Schriftenreihe, 1971, 21, 165-168.

Grabda-Kazubska B. Studies on abbreviation of the life cycle in Opisthioglyphe ranae (Fröhlich, 1791) and O. rastellus (Olsson, 1876) (Trematoda: Plagiorchiidae). Acta Parasitologica Polonica, 1969, 16 (27), 249-269.

Grabda-Kazubska B., Bayssade-Dufour C. \& Kiseliene V. Chaetotaxy and excretory system of Echinocercaria choanophila U. Szidat, 1936, a larval form of Cathaemasia hians (Rud., 1809) (Trematoda, Cathemasiidae). Acta Parasitologica Polonica, 35, 1990, 97-105.

GRABDA-KAZUBSKA B. \& LASKOWSKI Z. On the morphology and chaetotaxy of rediae and cercariae of Isthmiophora melis (Schrank, 1788) (Trematoda, Echinostomatidae). Acta Parasitologica, 1996, 41 (1), 7-12.

Hertel J., Holweg A., Haberl B., Kalbe M. \& HaAs W. Snail odour-clouds: spreading and contribution to the transmission success of Trichobilharzia ocellata (Trematoda, Digenea) miracidia. Oecologia, 2006, 147 (1), 173-180.

Horák P. \& KoláŘová L. Molluscan and vertebrate immune responses to bird schistosomes Parasite Immunology, 2005, 27 (7-8), 247-255.

JACKIEWICZ M. Lymnaeids of Europe (Gastropoda: Pulmonata:Lymnaeidae). Wydavnictwo Kontekst, Poznań, 2000 (in Polish).

Jones A., Bray R.A. \& Gibson D.I. Keys to the Trematoda. Volume 2. CABI Publishing Wallingford \& Natural History Museum, London, 2005.

Kalbe M., Haberl B. \& HaAs W. Snail host finding by Fasciola hepatica and Trichobilharzia ocellata: Compound analysis of "miracidia-attracting glycoproteins". Experimental Parasitology, 2000, 96 (4), 231-242.

Kanev I., Eisenhut U., Ostrowski de NúÑEZ M., Manga GonZALES M.Y. \& RAdev V. Penetration and paraoesophageal gland cells in Echinostoma revolum cercariae from its type locality. Helminthologia, 1993, 30, 131-133.

KHAN D. Studies on larval trematodes infecting freshwater snails in London (U.K.) and some adjoining areas. Part. I. Echinostome cercariae. Journal of Helminthology, 1960, 3/4, 277-304 
Kolářová L. \& HorÁK P. Morphology and chaetotaxy of Trichobilharzia szidati Neuhaus, 1952 cercariae (Trematoda: Schistosomatidae: Bilharziellinae). Helminthologia, 1996, 33, 3-7.

Kolářová L. \& HorÁk P. \& FajFrlík K. Cercariae of Trichobilharzia szidati Neuhaus, 1952 (Trematoda: Schistosomatidae): The causative agent of cercarial dermatitis in Bohemia and Moravia. Folia Parasitologica, 1992, 36, 399-400.

KoláŘOvÁ L. \& HoráK P. \& SitKO J. Cercarial dermatitis in focus: Schistosomes in the Czech Republic. Helminthologia, 1997, 34, 127-139.

Konecny R., Dvořák J., Horák P., \& Sattmann H. Zerkarien von Süßwasserschnecken in Ost-Österreich. Mitteilungen der Österreichischen Gesellschaft für Tropenmedizin und Parasitologie, 1999, 21, 77-82.

KÖNIG O. Cercarien von Trichobilharzia Skrjabin et Zakhakov, 1920 (Trematoda) in einem Baggersee in Bayern als Erzeuger von Bade-Dermatitis. Lauterbornia, 2001, 41, 33-43.

La Valette St. George A. Symbolae ad Trematodum evolutionis historiam. Dissertation, Berlin, 1855.

LOY C. \& HAAS W. Prevalence of cercariae from Lymnaea stagnalis snails in a pond system in South Germany. Parasitology Research, 2001, 87, 878-882.

LÜHE M. Parasitische Plattwürmer. In: Bauer A. (ed) Süßwasserfauna Deutschlands. Gustav Fischer, Jena, 1909.

NAŠInCOVÁ V. Trematode developmental stages in Czech aquatic snails and life-cycles of selected species of the family Omphalometridae and Echinostomatidae. PhD Thesis, Institute of Parasitology, Czechoslovak Academy of Sciences, České Budějovice, 1992, 268 p (in Czech).

NAŠInCOVÁ V., Bušta J. \& Krasnolobova T.A. Contribution to the developmental cycle and taxonomy of Neoglyphe sobolevi Shaldybin, 1953 (Trematoda, Omphalometridae). Folia Parasitologica, 1989, 36, 313-319.

Našincová V., Scholz T., \& Moravec F. The life cycle of Paryphostomum radiatum (Dujardin, 1845) (Trematoda: Echinostomatidae), a parasite of cormorants. Folia Parasitologica, 1993, 40, 193-201.

NeuHaus W. Biologie und Entwicklung von Trichobilharzia szidati n. sp. (Trematoda, Schistosomatidae), einem Erreger von Dermatitis beim Menschen. Zeitschrift für Parasitenkunde, 1952, 15, 203-266.

Nezvalová J. [Contribution to the knowledge of cercariae from South Moravia.] Spisy PřF UJEP v Brně, 1970, 515, 217-252. (in Czech)

NiEWIADOMSKA K. Verification of the life-cycles of Diplostomum spathaceum (Rudolphi, 1819) and D. pseudospathaceum Niewiadomska, 1984 (Trematoda, Diplostomidae). Systematic Parasitology, 1986, 8, 23-31.

NiewIADOMSKA K. \& LASKOWsKI Z. Systematic relationships among six species of Diplostomum Nordmann, 1832 (Digenea) based on morphological and molecular data. Acta Parasitologica, 2002, 47 (1), 20-28.

Niewiadomska K., Valtonen E.T. \& Siddal R. Cercariae from Lymnaea stagnalis in lake Kuuhankavesi (Central Finland). Acta Parasitologica, 1997, 42 (3), 132-137.

NitzsCH C.L. Beitrag zur Infusorienkunde oder Naturbeschreibung der Zerkarien und Bazillarien. Neue Schriften der Naturforschenden Gesellschaft zu Halle, 1817, 3, 1-123.

OdenING K. Furcocercarien (Trematoda: Strigeata und Schistomata, larvae) aus Brandenburg und Sachsen. Monatsberichte der Deutschen Akademie der Wissenschaften, 1962a, 4 (6), 384-392.

ODENING K. Notizen über Xiphidiozerkarien (Trematoda: Plagiorchiata, larvae) aus Brandenburg und Sachsen. Monatsberichte der Deutschen Akademie der Wissenschaften, 1962b, 4 (5), 300-311.

Odening K. Drei neue Furcocercarien aus dem Raum Berlin. Monatsberichte der Deutschen Akademie der Wissenschaften, 1964a, 6 (10), 739-743.

ODENING K. Die Entwicklungszyklen einiger Trematodenarten des Bleßhuhns Fulica a. atra im Raum Berlin. Biogische Rundschau, 1964b, 2 (3), 129-131.

Odening K. Die Altrices-Wirte einiger einheimischer Hausund Nutztiertrematoden. Bemerkungen zum tatsächlichen gegenwärtigen Stand der Kenntnisse. Angewandte Parasitologie, 1965a, 6 (2), 84-94.

ODENING K. Eine echinostome Cercarie mit 29 Kragenstacheln aus Gewässern bei Berlin und die Entwicklung ihres Exkretionssystems. Monatsberichte der Deutschen Akademie der Wissenschaften, 1965b, 7 (10/11), 818-822.

Opravilová V. Zur Kenntnis der Larvenstadien zweier Arten der Gattung Cotylurus Szidat, 1928. Folia Facultatis Scientiarum Naturalium Universitatis Purkynianae Brunensis, 1969, 10 (25), 93-106.

Pagenstecher H.A. Trematodenlarven und Trematoden. Helminthologischer Beitrag. Academische Verlagshandlung von J.C.B. Mohr, Heidelberg, 56 p.

PALM V. Die Zerkarienfauna der Süßwasserschnecken aus dem Gebiet bei Kleinmachnow bei Potsdam. Teil I. Furkozerkarien. Angewandte Parasitologie, 1966a, 7 (1), 1-16.

PALM V. Die Zerkarienfauna der Süßwasserschnecken aus dem Gebiet von Kleinmachnow bei Potsdam Teil II. Xiphidiozerkarien. Angewandte Parasitologie, 1966b, 7, 81-97.

Palm V. Die Zerkarienfauna der Süßwasserschnecken aus dem Gebiet von Kleinmachnow bei Potsdam. Teil III. Monostome Zerkarien (Notocotylidae), gymnozephale Zerkarien und Zerkariaeen. Angewandte Parasitologie, 1967, 8 (1), 7-21.

Pilz J., Eisele S. \& Disko R. Cercarial dermatitis (swimmer's itch). Case report of cercarial dermatitis caused by Trichobilharzia. Hautarzt, 1995, 46, 335-338.

Rudolfová J., Hampl V., Bayssade-Dufour C., Lockyer A.E., LITTLEWOOD D.T.J. \& HorÁK P. Validity reassessment of Trichobilharzia species using Lymnaea stagnalis as the intermediate host. Parasitology Research, 2005, 95, 79-89.

Samnaliev P., Dimitrov V. \& Bušta J. Argentophilic integumentary structures of Plagiorchis laricola Skrjabin, 1924 cercariae. Folia Parasitologica, 1983, 30, 329-334.

Sattmann H., Hörweg C. \& Konecny R. Zerkariendermatitis in Österreich - Rückblick und Perspektiven. Denisia, 2004, 13, 457-461.

SCHEURING L. Der Lebenszyklus von Sanguinicola inermis Plehn. Zoologische Jabrbücher. Abteilung für Anatomie, 1922, 44, 265-310. 
STYCZYŃSKA-JuREwicz E. Expansion of cercariae of Diplostomum spathaceum Rud., 1819, a common parasite of fishes, in the littoral zone of the lake. Polskie Archiwum bydrobiologii, 1959, 6 (19), 105-116.

STYCZYŃSKA-JuREWICZ E. [Relation between negative geotaxis of cercariae of Opisthioglyphe ranae Duj. and the oxygen conditions in small water bodies.] Wiadomosci Parazytologiczne, 1961, 7 (2 Suppl.), S195-S197 (in Polish).

SudARIKOV V.E. [Order Strigeidida (La Rue, 1926) Sudarikov, 1959. Part 1. Morphological characteristics of strigeids and superfamily Strigeoidea Railliet, 1919.] In Skryabin K.I. (ed.) [Trematodes of animals and man.] Osnovy trematodologii, 1959, 16, 217-631 (in Russian).

Vojtek J. \& VojTKová L. Life cycles of trematodes of the order Strigeidida in Czechoslovakia. Scripta Facultatis Scientiarum Naturalium UJEP Brno, 1971, 1 (4), 303-312.

WISNIEWSKI L.W. Characterization of the parasitofauna of an eutrophic lake. Acta Parasitologica Polonica, 1958, 6, 164.

ZAjíčEK D. [Cercariae and other developmnetal stages of trematodes in snails from some fishpond systems in South Bohemia.] Ceskoslovenská Parasitologie, 1963, 10, 187-205 (in Czech).

ZAJíčEK D. [On the epizootiology of trematodes of the genus Apatemon Szidat, 1928 and Cotylurus Szidat, 1928 (Trematoda: Strigeidae) in ducks from south bohemian fishpond systems.] Veterinární Medicína, 1971, 16 (44), 5360 (in Czech).

ZAJÍČEK D. \& VALENTA Z. [Contribution to the occurrence of furcocercariae in some areas of Bohemia.] Československá Parasitologie, 1964, 11, 273-293 (in Czech).

ŻBIKOwska E. Is there a potential danger of "swimmer's itch" in Poland? Parasitology Research, 2003, 89 (1), 59-62.

ŻBIKOWsKA E. Infection of snails with bird schistosomes and the threat of swimmer's itch in selected Polish lakes. Parasitology Research, 2004, 92, 30-35.

ŽĎÁRSKÁ Z. ČSSR [Larval stages of trematodes from aquatic snails from the territory of ČSSR.] Československá Parasitologie, 1963, 10, 207-262 (in Czech).

ŽĎÁrská Z. [On the problem of development of some trematodes.] Československá Parasitologie, 1964a, 11, 295-308 (in Czech).

ŽĎÁRSKÁ Z. Further findings of larval trematodes in molluscs from Czechoslovakia. Acta Societatis Zoologicae Bohemoslovenicae, 1964b, 28 (1), 14-25.

ŽĎÁRSKÁ Z. Der Entwicklungszyklus des Trematoden Plagiorchis laricola (Skrjabin, 1924). Acta Societatis Zoologicae Bohemoslovenicae, 1966, 20 (2), 179-184.

Reçu le 11 septembre 2006 Accepté le 12 décembre 2006 